\title{
Consistent Results with the Rotaglide Total Knee Arthroplasty. A 2-5 Year Follow Up
}

\author{
Christopher Wilson* \\ Department of Orthopedic surgery, Flinders Medical Centre and Finders University, Australia
}

\begin{abstract}
Aims: In this study we present the outcomes for patients with the rotaglide mobile meniscal knee prosthesis. All patients reviewed had this prosthesis implanted as a primary total knee Arthroplasty in one local teaching hospital dept. The minimum follows up period was 2 years (range 2-8.2).

Method: Patients were assessed clinically by the junior author (CW) and results were standardised using the Hospital for Specialist Surgery (HSS) knee score. Complications were quantified from patient history and examination of the case notes.

Results: Two hundred and two knees were reviewed. The results were then consolidated into groups with a minimum follow up of 2,3,4 and 5 years. The average HSS score was consistent at 91 for all four groups. Complications were also consistent ranging between 10 and 11\% in all groups. These are summarised in Table 1 below. The commonest complication was superficial wound infection (4.95\%). There were also three deep infections (1.49\%) and two revisions due to meniscal failure (0.99\%).

Conclusion: These results suggest the rotaglide total knee Arthroplasty offers safe and effective treatment with constantly good clinical results at 2-5 years follow up. The complication rate was also consistent over this period with a low incidence of meniscal failure and deep infection. There was no difference in outcome in knees with or without patellar resurfacing. There have been no failures due to aseptic loosening in this group to date.
\end{abstract}

KEYWORDS: Consistent; Knee; Arthroplasty; rotaglide \& Outcomes

Table 1: The relative incidence of complications for knees in each follow up group. Relative percentages shown in brackets.

\begin{tabular}{|c|c|c|c|c|}
\hline Complications & $\mathbf{5}$ yrs. & $\mathbf{4}$ yrs. & $\mathbf{3}$ yrs. & $\mathbf{2}$ yrs. \\
\hline Wound infection & $3(3.2)$ & $7(4.9)$ & $9(5.9)$ & $2(1.1)$ \\
\hline Deep infection & Nil & $1(0.7)$ & $2(1.1)$ & $3(1.5)$ \\
\hline Deep venous thrombosis & $2(2.2)$ & $2(1.4)$ & $2(1.1)$ & $2(1.0)$ \\
\hline Meniscal failure & $2(2.2)$ & $2(1.4)$ & Nil & $2(1.0)$ \\
\hline Wound haematoma & Nil & Nil & $1(0.6)$ & $1(0.5)$ \\
\hline Patellar revision & $1(1.1)$ & $1(0.7)$ & $1(0.6)$ & $1(0.5)$ \\
\hline Proximal tibial\# & $1(1.1)$ & $1(0.7)$ & $1(0.6)$ & $1(0.5)$ \\
\hline Pulmonary embolism & $1(1.1)$ & Nil & $1(0.6)$ & $1(0.5)$ \\
\hline Heterotopic bone formation & Nil & $15(10.5)$ & $19(10.5)$ & $1(0.5)$ \\
\hline Total & $10(10.7)$ & & & $22(10.9)$ \\
\hline
\end{tabular}

Quick Response Code:

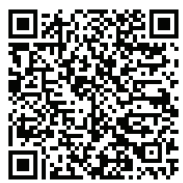

Address for correspondence: Christopher Wilson, Department of Orthopedics Level 5, Flinders Medical Centre Flinders Drive, South Australia

Received: September 24, $2021 \quad$ Published: October 06, 2021

How to cite this article: Christopher W. Consistent Results with the Rotaglide Total Knee Arthroplasty. A 2-5 Year Follow Up. 2021- 3(5) OAJBS.ID.000328. DOI: 10.38125/OAJBS.000328 


\section{INTRODUCTION}

In this study we present the outcome for patients with the rotaglide mobile meniscal knee prosthesis. All patients reviewed had this prosthesis implanted as a primary total knee for the diagnosis of osteoarthritis. This unit provides a trauma and elective service in a busy District General hospital setting. Surgery was performed by one of two experienced Consultants in our center or by a trainee under their supervision. A standard medial para-patellar approach was used, and the components fixed using Palacos cement with Gentamycin. Patellar resurfacing was performed at the preference of the operating consultant.

The rotaglide total knee Arthroplasty (Corin Medical) has been implanted in our centre for 8 years. This three-part mobile meniscus prosthesis allows the polyethylene meniscal component

to move in two planes. AP translation of $5 \mathrm{~mm}$ is allowed combined with rotation 12.5 degrees on either side of the midline. Two metal pegs on the tibial tray prevent excessive movement of the meniscal component. The condyles of the femoral component are based on the surface of a sphere.

This increases the contact area between the femoral component and the meniscal insert throughout flexion [1,2]. Increased congruity may reduce production of polyethylene wear debris and therefore reduce aseptic loosening of the cemented components [3]. The inferior surface of the meniscal insert and articulating surface of the tibial baseplate are flat and therefore highly congruent. Movement between these components in the normal gait cycle may reduce stress at the cement / bone interface [4].

In our previous review the rotaglide prosthesis was found to offer a good clinical outcome for patients at 5 years minimum follow up with a low level of complications [5]. However, the number of patients involved was small (73 knees). The aim of this study was to assess the outcomes of a larger patient cohort and to follow up the clinical knee scores and complication rates over a number of years. This allows us to follow the progression of our results through our learning curve with the rotaglide prosthesis. We aim to show that consistent and reproducible results can be obtained within the setting of a busy District General Hospital.

\section{METHODS}

Patients who underwent primary total knee Arthroplasty were identified using the hospital diagnosis coding system and case notes pulled for examination. All patients who had the rotaglide prosthesis implanted with a minimum follow up of 2 years were included. The assessment of patients consisted of a formal clinical examination and a functional questionnaire. Patients were assessed clinically by the author (CW) and results were standardised using the Hospital for Specialist Surgery (HSS) knee score. Complications were quantified from patient history and examination of the case notes. Functional scoring and clinical examination findings were recorded on data sheets standardised using the Hospital for Specialist Surgery (HSS) Knee score [6]. Complications were assessed using data from patient interviews and review of the case notes.

Results were compiled with specific reference to all knees with a minimum follow up of 5,4,3 \& 2 years respectively. These are summarised later in table or graph formats. Specific attention was paid to clinical \& functional scoring. Patellar resurfacing was also assessed. One of our senior consultants routinely resurfaces while one does not. This allowed us to compare the HSS scores separately for both of these groups. Patellar resurfacing was performed in $48 \%$ of cases. Range of movement obtained and any complications that occurred were also subdivided into the 4 subgroups above.

\section{RESULTS}

On review of patient's case records of 274 knees in total 225 knees were implanted in patients within the above inclusion criteria (82.1\%). Of this Group 17 had died (7.6\%) and 6 patients were lost to follow up (2.7\%). Two hundred and two knees were therefore reviewed. The results were then consolidated into groups with a minimum follow up of 5,4,3 and 2 years respectively. These results are summarised in below.

In our first group with a minimum of 5 years, 93 knees were reviewed. 4 patients had died (4.3\%) and 3 were lost to follow up (3.2\%). The average HSS score was 91.1 with only 5 patients scoring less than 85 (5.4\%). When patients were divided into two groups with reference to patellar resurfacing there was no significant difference in HSS scoring. The Patellar resurfacing group (59 knees) had and average HSS score of 91.4 with the non-resurfaced group (34 knees) averaging 90.4. The average Range of movement (ROM) in this patient group overall was 101.1 degrees with a range of 70-125 degrees. Complications have been summarised in Table 1 below.

In our second sub-group the minimum follows up period was 4 years, and 143 knees were reviewed. 7 were lost as the patients had died (4.9\%) and 4 were lost to follow up (2.8\%). The average HSS score was 91.7 with only 6 knees scoring less than 85 (4.2\%). In the patellar resurfacing group (83 knees) the HSS score was (91.7) and in the non-resurfacing group (60 knees) 91.6. The average ROM was 101.9 degrees with a range of 60-130 degrees. The complications in this sub-group have been summarized in Table 1 below.

In our third patient sub-group the minimum follows up time was reduced to 3 years with 181 knees reviewed. In this group 19 knee were lost, 15 because the patients had died (8.3\%) and 5 were lost to follow up (2.8\%). The average HSS for this group was 91.6 with 9 knees scoring less than 85 (4.9\%). HSS scores were 91.5 for the patellar resurfacing group (103 knees) and 91.7 for the nonresurfacing group (78 knees) respectively. All complications have been summarized in Table 1 below.

In the fourth and last patient sub-group the minimum follows up time was only two years. In this group 202 knee were reviewed. 17 knees were lost as the patients had died (7.6\%) and 7 were lost to follow up. The HSS score in this group remained static at 91.7 with 12 knees scoring less than 85 (5.9\%). The scores for the patellar resurfacing and non-resurfacing groups were 91.6 in 105 knees and 91.8 in 97 knees respectively. Average ROM was 101.9 degrees with a range of 60-130 degrees. The final summary of complications for this group is outlined in Table 1 below.

\section{DISCUSSION}

Results from our previous report suggest that the rotaglide Total Knee Arthroplasty offers patients a good functional outcome with a low level of Complications. This paper was based on a small series of 74 knees with a minimum follow up of 5 years (5). In this series we now have results over 5 years for 93 knees with an average HSS score of 91.1 . Also $95.8 \%$ of patients scored over 85 on the HSS score which is defined as an excellent clinical outcome [6]. One additional superficial wound infection occurred 
in these additional 19 knees. It was suggested in this paper that the meniscal complications, one meniscal fracture and one meniscal dislocation were due to problems with surgical technique early in the surgeons learning curve. In this series can compare the results of over 200 knees implanted in our hospital by two surgeons over a period of 8 years. This allows comparison of functional results and complications over a period of time. For simplicity the patients have been divided in to four sub-groups with a minimum follow up time of 5,4,3 \& 2 years respectively. With reference to functional outcomes the HSS scores of patients over the 4 sub-groups have been plotted with results for all patients, patients with and without patellar resurfacing respectively. These is no significant variation in these values over time. These are shown in Figure 1 below. There was no significant change in the Range of movement for patents in all 4 groups with a range of average values from 101.1 to 101.9 degrees (range from 60 -130 degrees). These results are summarized in Figure 2 below.

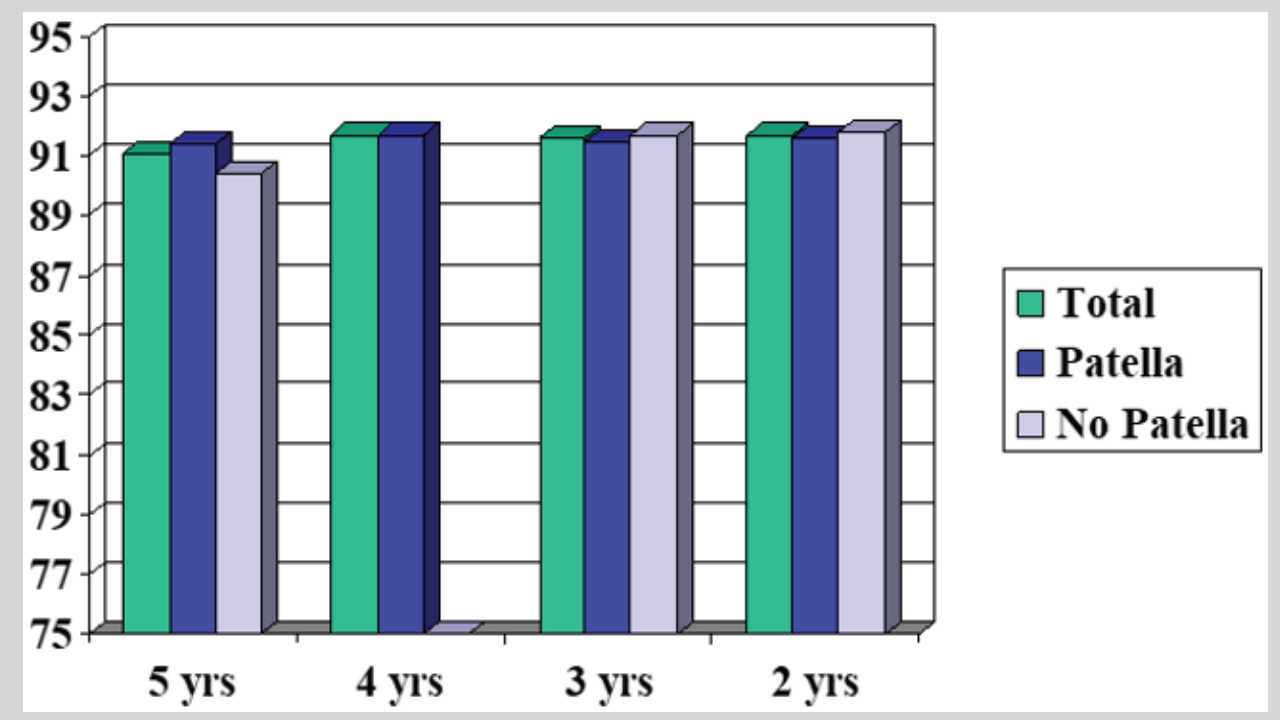

Figure 1: Relative HSS values over time for all patients, and patients with and without patellar resurfacing.

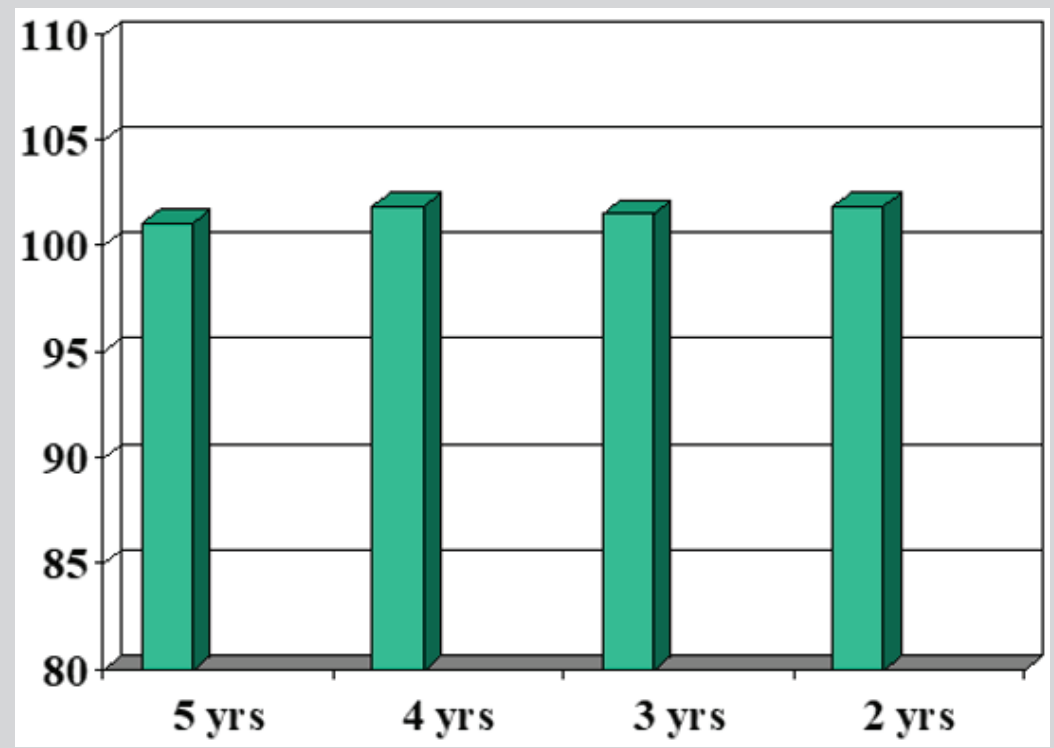

Figure 2: Average range of movement in degrees for each patient subgroup.

With reference to complications these have been summarized in the above Table 1. There was no significant change in the incidence of total complications varying from $10.5-10.9 \%$. The commonest complication is superficial wound infection defined as erythema and wound discharge treated successfully with anti-biotics. The incidence of this complication varied from 3.2-5.9\% over all 4 patient sub- groups. Deep infections varied from $0 \%$ in the 5 years follow up group to $1.5 \%$ with a follow up of min. 2 years. In total there were three deep infections. Two were treated with staged revision surgery. The third knee has responded well to date with open surgical debridement and Intra-venous antibiotics. Other complications were low in number and there was no significant change over the 4 sub-groups. The trends in complication rates are summarized below in Figure 3. For Clarity we have included Total complications, superficial infections, deep infections and meniscal complications.

These results suggest that there is no significant change in function results or overall complications with rotaglide knee arthroplasty if results are followed up over several years. As the 
minimum follow up time falls in these sub-groups the number of patients increases. However, these groups also accumulate patients implanted later in our series. Therefore, the absence of significant change in HSS scoring or range of movement values as we progress through the patient sub-groups suggests that the performance of our primary rotaglide total knee arthroplasties has remained consistent over time. Our experience with this prosthesis is now established for over a decade. The average HSS scoring is almost unchanged over this time with very few patients scoring less than 85 . Scores over this level have previously been defined as an indicator of an excellent clinical outcome [6]. There was also no significant difference in the scoring between patients who had undergone patellar resurfacing or not. Only one patient from the unsurfaced group underwent revision surgery to resurface the patella due to knee pain. Although opinions obviously vary it has been suggested in recent reports that there is reasonable doubt over whether routine resurfacing of the patella is required [7]. In this particular Prosthesis this particular question requires further analysis with work directed at patellar resurfacing. When comparing the Range of motion obtained the average was over 100 degs in all groups. The Tibial cut with this prosthesis is designed with a 4-degree backward tilt to try and increase the amount of flexion obtained. There was no significant variation in the ROM values for each subgroup ranging from 101.1 to 101.9 degrees. Using ROM as an outcome measure for this prosthesis again our results are consistent over time.

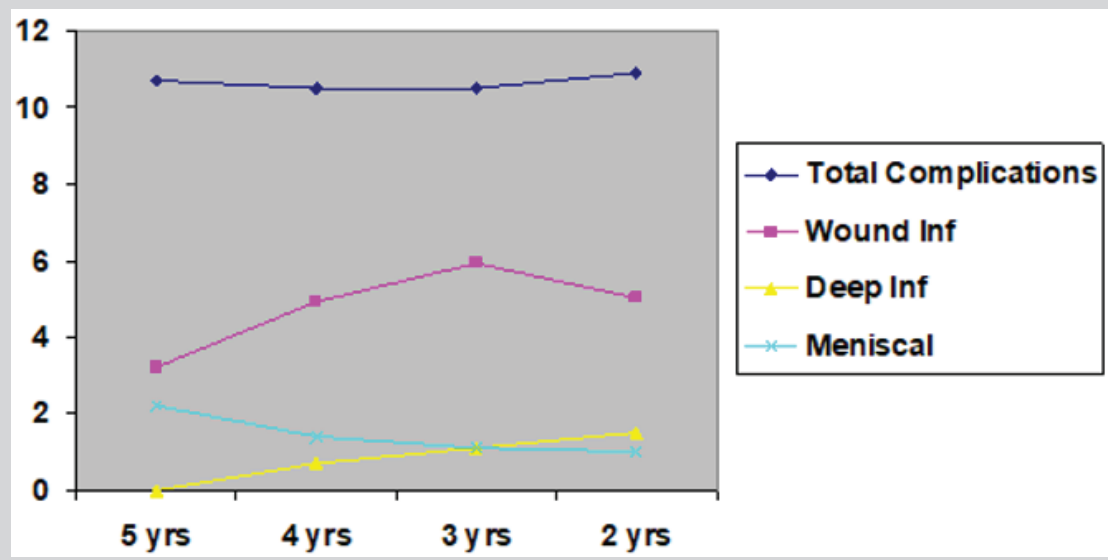

Figure 3: The relative incidences of complications for each patient sub-group.

When comparing the incidence of specific complications, the commonest by far was superficial wound infection. The incidence in our 5 years follow up group was $3.2 \%$. However, the incidence in all other groups was nearer $5 \%$. It is difficult to speculate whether this change is due to a specific problem leading to slightly more infection or if the smaller 5-year group simply had slightly less. Other complications are less affected, and their numbers are smaller making inferences less powerful. However, with note to thromboembolic complications these are defined as proven DVT or pulmonary embolism in the post operative period both in the Orthopedic department and medical department. It is likely that some patients will have sustained sub clinical venous thrombotic events that were never investigated.

The Deep infection rate rose slowly over the 4 patient groups. The numbers are however low and this trend is probably just due to accumulation of patients. There are insufficient cases to produce a clinically significant change. For all patients available for review the deep infection rate was $1.49 \%$. On review of the case notes of patients lost to follow up and the available notes of those who died there were no other deep infections. This gives us a possible deep infection rate as low as $1.33 \%$. There were two cases of meniscal complications in the 1st patient sub-group, however in subsequent groups there have been no cases. This supports the inference in our previous report that correction of surgical technique with reference to soft tissue balancing can help prevent such problems.

\section{CONCLUSION}

The rotaglide prosthesis has been used by other centers in the UK. However, few results have been published yet concerning its performance [8-10]. These results suggest the rotaglide total knee Arthroplasty offers safe and effective treatment for osteoarthritis with consistent good clinical results. In this series follow up was subdivided into 4 specific sub-groups. Patient's results were recorded in groups with a minimum follow up of 5,4,3 and 2 years respectively. As you move from one group to the next the minimum follow up time falls. However, the total number of patients increases. You are also moving along the learning curve of our center with the rotaglide prosthesis. The clinical outcomes of patients were unchanged throughout these groups. Patient's performance with this implant has not therefore significantly improved or worsened over time.

There seems to be no difference in outcome between patients with and without resurfacing of the patella. The overall complication rate was also consistent over this period and when attention is paid to significant complications there was little change. The one exception being two meniscal complications early in our series. Our current series is now over 300 knees, and their outcomes remain under review. We conclude that with our center we offer our patients a safe, consistent and effective service for patients with osteoarthritis of the knee. Patients implanted with the rotaglide Total Knee Arthroplasty have good functional outcomes. The complication rates are low and have remained constant as our series increases.

\section{REFERENCES}

1. Brooks S. The rotaglide knee, the solihull experience at nine years follow up. Data supplied by Corin Medical Ltd.

2. Polyzoides AJ (1999) Design characteristics, experimental studies and ten years of clinical experience with fully conforming mobile bearing knee prosthesis. proceedings of the international symposium of the knee.

3. JJ Callaghan (2001) Mobile-bearing knee replacement: Concepts and results. AAOS Instructional Course Lectures 50: 431-449. 
4. Tsakonas A, Polyzoides AJ (1997) Reduction of polyethylene in a congruent meniscal knee prosthesis. Acta Orthop Scand 275: 127-131.

5. Wilson CJ, Tait G (2003) Five-year review of the Rotaglide total knee Arthroplasty. The Knee 10(2): 167-171.

6. Insall JN (1989) Rational of the Knee Society clinical rating system. Clin Orthop 248: 13-14.

7. Wood DJ, Smith AJ (2002) Patellar resurfacing in total knee arthroplasty: A prospective, randomised trial. J Bone Joint Surg Am 85(2): 212-217.
8. Polyzoides AJ (1996) The rotaglide total knee arthroplasty. J Arthroplasty 11: 453-459.

9. Crova M, Olivero C (2000) Rotating knee prosthesis, surface or hinge replacement? Orthopade 29: 543-544.

10. Polyzoides AJ, Tsakonas A (1993) The rotaglide knee replacement: Results of the first 311 cases. proceedings of the rotaglide total knee course. 